\title{
Editorials
}

\section{Post-thoracotomy shoulder pain and gabapentin: a tale of two enigmas}

Peter MacDougall PhD MD FRCPC, * Peter Slinger MD FRCPC $\dagger$

I $\mathrm{N}$ this issue of the Journal, Huot et al. ${ }^{1}$ report the findings of a well designed, placebo-controlled, randomized trial, evaluating preoperative gabapentin for prevention of post-thoracotomy shoulder pain (SP). These investigators premedicated patients with gabapentin $1200 \mathrm{mg}$ po, two hours prior to thoracotomy, with thoracic epidural analgesia in situ, for management of postoperative incisional pain. Unfortunately, gabapentin, effective as an analgesic in many postoperative states, did not appear to be effective in preventing post-thoracotomy SP. The authors note that, while there was no improvement in relief of pain in the gabapentin group, there was an increase in sedation as a side effect of gabapentin. This report brings to light the convergence of two enigmas in postoperative pain management: the mechanism of action of gabapentin and the phenomenon of SP after chest surgery.

\section{Postoperative SP}

Postoperative SP is common after surgery involving direct trauma to the phrenic nerve or indirect irritation of the phrenic nerve via its diaphragmatic fibres. Postoperative SP is also common after a number of laparoscopic procedures., ${ }^{2,3}$ Rates of SP after laparoscopic cholecystectomy may be as high as $80 \%{ }^{2}$ Maneuvers designed to reduce abdominal inflation pressure, to minimize temperature variations with the insufflating gas, or to reduce the possible production of carbonic acid from $\mathrm{CO}_{2}$ have achieved variable success in reducing postoperative, laparoscopic SP. ${ }^{2,3}$ While direct evidence is lacking, the phrenic nerve has been postulated to act as the mediator for postoperative SP following laparoscopic procedures. ${ }^{2}$
Postoperative SP after thoracic surgery usually becomes manifest in the recovery room. The incidence has been reported to range from $21 \%$ to $97 \%$ of patients having chest surgery. ${ }^{4,5}$ The pain is classically described as moderate to severe, aching, over the deltoid or the superior/posterior aspect of the shoulder, or over the lateral $1 / 3$ of the clavicle ipsilateral to the surgery. ${ }^{6}$ While current evidence remains limited, there is strong suggestion that the phrenic nerve is responsible for this classic presentation of post-thoracotomy SP. Regional techniques using interscalene or stellate ganglion block have relieved post-thoracotomy SP. Case reports concerning direct blockade of the phrenic nerve $\mathrm{e}^{7}$ as well as randomized controlled trials of local anesthetic infiltration of the phrenic nerve, ${ }^{8,9}$ have been shown effective in reducing post-thoracotomy SP. Barak et al. ${ }^{10}$ conducted a randomized controlled study comparing interscalene block $v s$ intravenous diclofenac, for relief of postthoracotomy SP, and demonstrated rapid relief of post-thoracotomy SP in the interscalene block group. Given that these regional techniques are associated with high levels of phrenic nerve anesthesia, it is probable that this was the mechanism of the pain relief.

The natural history of post-thoracotomy SP has not been fully studied, and much of what we know of it is gleaned from case reports. Bussieres et al. ${ }^{4}$ and MacDougall et al. ${ }^{11}$ have demonstrated that, in addition to the high rate of postoperative SP after open thoracotomy, patients undergoing less invasive thoracoscopy or video-assisted thoracoscopy (VATS) procedures also had a high incidence of postoperative SP. Bussieres et al. ${ }^{4}$ reported that $21 \%$ of patients having thoracotomy or thoracoscopy experience SP.

CAN J ANESTH $2008 / 55: 6 /$ pp 323-327

From the Departments of Anesthesia, Dalhousie University, ${ }^{*}$ Halifax, Nova Scotia; and the University of Toronto, $†$ Toronto, Ontario, Canada.

Address correspondence to: Dr. Peter Slinger, Department of Anesthesia, The Toronto General Hospital, 200 Elizabeth Street, Toronto, Ontario M5G 2C4, Canada. Phone: 416-340-5164; Fax: 416-340-3698; E-mail: peter.slinger@uhn.on.ca Competing interests: None declared. 
MacDougall et al. ${ }^{11}$ studied 41 patients undergoing VATS or thoracotomy for lung or esophageal surgery. Post-thoracotomy SP occurred at an overall rate of $64.6 \%$. Post-VATS SP occurred in $70 \%$ of patients. After accounting for atypical presentations, the rates of post-thoracotomy SP and post-VATS SP declined to $48.4 \%$ and $40 \%$, respectively.

In the same study, MacDougall et al. ${ }^{11}$ noted a number of atypical presentations of SP, suggesting that there are additional causes of post-thoracotomy and post-VATS SP. In the thoracotomy group, some patients experienced SP after a walk, while others experienced anterior chest pain and SP in the postanesthesia care unit. Further manifestations included pain over the neck and scapula and pain over the upper scapula at the proximal end of the thoracotomy incision. Amongst patients reporting pain in the VATS group, two reported scapular pain and one reported pain similar to his known arthritis. Anecdotal evidence suggests that extension of the posterior, chest drain to the apical pleura may also cause shoulder tip pain, which can be relieved by withdrawal of the chest drain by a few centimetres (Slinger P, and McRae K, personal communication). These findings are consistent with other case reports. ${ }^{7}$ As well, while Scawn et al. ${ }^{8}$ and Danelli et al. ${ }^{9}$ demonstrated a significant reduction in post-thoracotomy SP, following injection of local anesthetic into the peri-phrenic nerve fat pad, a significant number of their patients continued to experience post-thoracotomy SP. Therefore, there is suggestion that post-thoracotomy $S P$, in a subset of patients, may originate from sources other than the phrenic nerve. Possible causes include: pre-existing arthritic conditions; patient positioning; glenohumeral or acromioclavicular joint strain; muscle strain over the scapula and trapezius; extension of the incision beyond the level of the epidural blockade; or chest drain irritation of the parietal pleura.

Despite the intense and distressing nature of this postoperative pain syndrome, it appears to be of limited duration. Post-thoracotomy SP has been reported to decline in the first four to six hours. ${ }^{11}$ Barak et al. ${ }^{10}$ describe gradually declining post-thoracotomy SP extending to at least postoperative day one, and Mac et al. ${ }^{5}$ describe gradually declining pain scores for $48 \mathrm{hr}$ after surgery. MacDougall et al. ${ }^{11}$ followed patients until the pain resolved, or until hospital discharge. Both the intensity and the duration of the pain declined steadily in the postoperative period. These investigators observed that the average duration of post-thoracotomy SP was four days, and the average duration of post-VATS SP was less than three days. ${ }^{11}$ No patient had post-thoracotomy SP or post-VATS $\mathrm{SP}$ at the time of discharge from the hospital.

\section{Gabapentin and postoperative pain}

In recent years, gabapentin has been investigated as an agent to relieve postoperative pain and, perhaps, to prevent chronic postoperative pain. ${ }^{12,13}$ Perioperative gabapentin has been effective at reducing postoperative pain in orthopedic, gynecologic, urologic, breast, and ENT surgery. ${ }^{12,13}$ It has been shown to reduce postoperative pain, and to decrease dependence on opioids for postoperative pain control. ${ }^{12,13}$ Interestingly, preoperative gabapentin $(300 \mathrm{mg}$ po), a dose $1 / 3$ of that used by Huot et al., ${ }^{1}$ has been shown to be effective in reducing postoperative pain after laparoscopic cholecystectomy. ${ }^{14}$ However, in this large trial, the type of pain (incision/ visceral/ shoulder) was not described. In addition to acting as an anti-nociceptive agent, gabapentin reduces postoperative nausea and vomiting after laparoscopic cholecystectomy. ${ }^{12}$ It is unclear whether this effect is secondary to a decrease in opioid requirements or secondary to a direct effect on mediators such as tachykinin.

At present, the exact mechanism by which gabapentin functions to reduce acute pain is unknown. The drug inhibits $\mathrm{Ca}^{++}$channels in hyper-excited neurons, but spares normal neurons. ${ }^{12,13,15}$ Peng and Wijeysundera ${ }^{12}$ suggest that this inhibitor of the $\alpha-2-\delta$ subunit of calcium channels may be better termed an 'anti-sensitization' agent as well as an anti-nociceptive agent. Gabapentin binds specifically to the $\alpha-2-\delta$ subunit of the voltage-dependent calcium channel in neurologic tissue, which, in turn, has at least three further subunits $(\alpha-2-\delta-1, \alpha-2-\delta-2$, and $\alpha-2-\delta-3)$ with different binding affinities for gabapentin. ${ }^{15}$ It has a moderate effect on neurotransmitter release in neocortical cells and in peripheral nerves, ${ }^{15}$ and it has an effect on the modulation of a number of protein kinases involved in the induction and the maintenance of hyperalgesic states. ${ }^{15}$

In summary, patients having procedures leading to direct or indirect irritation of phrenic nerve fibres are affected by high rates of postoperative SP. This is especially true in patients having chest surgery, either traditional thoracotomy or VATS procedures. However, unlike many forms of postoperative pain, this pain does not seem to be decreased by pre-treatment with gabapentin. Further, while we understand the binding sites of gabapentin and some of its effects, both at a molecular and at a clinical level, many unanswered questions remain. Is the effect of gabapentin, in some postoperative pain states mediated by different subunits of the $\alpha-2-\delta$ subunit of the calcium channel? Is there a variation in the post-translational modulation of neurotransmitters among postoperative pain states? Does post-thoracotomy SP have any ele- 
ment of hyperalgesia or "wind-up" that is amenable to preemptive analgesia? The broad array of potential tissue-gabapentin interactions offers fertile ground for speculation as to the underlying mechanism by which gabapentin affects many postoperative pain states, but does not affect post-thoracotomy SP. Perhaps this "crossroads" of a unique postoperative pain state and a drug with novel mechanisms of action will eventually provide a window for clearer understanding of what makes us feel pain following surgery.

\section{La douleur à l'épaule à la suite d'une thoracotomie et la gabapentine : l'histoire de deux énigmes}

Dans ce numéro du Journal, Huot et coll. ${ }^{1}$ rapportent les résultats d'une étude randomisée et contrôlée par placebo bien conçue, dont l'objectif était d'évaluer l'administration préopératoire de gabapentine pour prévenir les douleurs à l'épaule post-thoracotomie. Les chercheurs ont administré 1200 mg de gabapentine oralement aux patients en guise de prémédication deux heures avant la thoracotomie, avec une analgésie péridurale thoracique in situ, pour la prise en charge de la douleur d'incision postopératoire. Malheureusement, la gabapentine, qui est efficace en tant qu'analgésique dans nombre d'états postopératoires, ne semble pas l'être pour prévenir la douleur à l'épaule à la suite d'une thoracotomie. Les auteurs remarquent que, alors qu'il n'y a pas eu d'amélioration du soulagement de la douleur dans le groupe gabapentine, une augmentation de la sédation a été observée comme effet secondaire de la gabapentine. Cette étude souligne la convergence de deux énigmes qui accompagnent la prise en charge de la douleur postopératoire : le mécanisme d'action de la gabapentine et le phénomène de douleur à l'épaule après une chirurgie thoracique.

\section{Douleur postopératoire à l'épaule}

La douleur postopératoire à l'épaule est fréquente après une chirurgie et implique une lésion directe ou une irritation indirecte du nerf phrénique par le biais de ses fibres diaphragmatiques. La douleur postopératoire à l'épaule est également fréquente après nombre d'interventions laparoscopiques. ${ }^{2,3}$ L'incidence de douleurs postopératoires à l'épaule après une cholécystectomie par laparoscopie peut atteindre $80 \% .^{2}$ Les mesures destinées à réduire la pression de gonflage abdominal, à minimiser les variations de température avec le gaz insufflé ou à réduire la production potentielle d'acide carbonique à partir du $\mathrm{CO}_{2}$ ont eu un succès variable dans la diminution de la douleur postopératoire laparoscopique à l'épaule. ${ }^{2,3}$ Bien que nous manquions de données probantes directes, il a été postulé que le nerf phrénique agit en tant que médiateur de la douleur postopératoire à l'épaule à la suite des procédures par laparoscopie. ${ }^{2}$

La douleur postopératoire à l'épaule à la suite d'une chirurgie thoracique se manifeste en général dans la salle de réveil. Son incidence va de $21 \%$ à $97 \%$ chez les patients subissant une chirurgie thoracique ${ }^{4,5} \mathrm{La}$ douleur est traditionnellement décrite comme modérée à sévère au site du deltoïde ou à l'emplacement supérieur / postérieur de l'épaule, ou s'étendant sur le tiers latéral de la clavicule ipsilatérale à la chirurgie. ${ }^{6}$ Quoique les données probantes actuelles soient limitées, plusieurs éléments laissent à penser que le nerf phrénique est à l'origine de cette présentation conventionnelle de la douleur postopératoire à l'épaule après une thoracotomie. Les techniques d'anesthésie régionale faisant usage de blocs interscalénique ou du ganglion stellaire ont soulagé les douleurs à l'épaule à la suite d'une thoracotomie. Des présentations de cas traitant de blocs directs du nerf phrénique ${ }^{7}$ ainsi que des études randomisées contrôlées d'infiltration d'anesthésiant local au site du nerf phrénique ${ }^{8,9}$ ont démontré leur efficacité pour réduire les douleurs à l'épaule post-thoracotomie. Barak et coll. ont mené une étude randomisée contrôlée comparant le bloc interscalénique au diclofénac intraveineux pour soulager la douleur à l'épaule post-thoracotomie et ont montré un soulagement rapide de la douleur à l'épaule post-thoracotomie dans le groupe du bloc interscalénique. Étant donné que ces techniques régionales sont associées à des niveaux élevés d'anesthésie du nerf phrénique, il est probable qu'il s'agissait là du mécanisme du soulagement de la douleur.

L'histoire naturelle de la douleur à l'épaule à la suite d'une thoracotomie n'a pas été étudiée en profondeur, et ce que nous en savons a pour la plupart été glané ici et là dans des présentations de cas. Bussières et coll. ${ }^{4}$ et MacDougall et coll. ${ }^{11}$ ont démontré qu'en plus de l'incidence élevée de douleur postopératoire à l'épaule après une thoracotomie ouverte, les patients 
subissant une thoracoscopie moins effractive ou une vidéo-thoracoscopie (VTS) présentaient également une incidence élevée de douleurs postopératoires à l'épaule. Bussières et coll. ${ }^{4}$ rapportent que $21 \%$ des patients subissant une thoracotomie ou une thoracoscopie ressentent des douleurs à l'épaule. MacDougall et coll. ${ }^{11}$ ont examiné 41 patients subissant une VTS ou une thoracotomie dans le cadre de chirurgie des poumons ou de l'œsophage. L'incidence globale de douleur à l'épaule post-thoracotomie a atteint 64,6\%. La douleur à l'épaule post-VTS est survenue chez 70 $\%$ des patients. Si l'on tient compte des présentations atypiques, la fréquence de douleur à l'épaule postthoracotomie et post-VTS a décru à $48,4 \%$ et $40 \%$, respectivement.

Dans la même étude, MacDougall et coll. ${ }^{11}$ ont observé plusieurs présentations atypiques de la douleur à l'épaule, suggérant qu'il existe d'autres causes de douleur à l'épaule post-thoracotomie et post-VTS. Dans le groupe thoracotomie, quelques patients ont ressenti une douleur à l'épaule après avoir marché, alors que les autres ont ressenti des douleurs thoraciques précordiales et à l'épaule à la salle de réveil. D'autres douleurs se sont manifestées au niveau du cou et de l'omoplate ou du haut de l'omoplate à l'extrémité proximale de l'incision de thoracotomie. Parmi les patients ayant ressenti des douleurs dans le groupe VTS, deux ont rapporté des douleurs à l'omoplate et un patient a ressenti une douleur semblable à son arthrite connue. Des données anecdotiques suggèrent qu'insérer le drain thoracique postérieur jusqu'à la plèvre apicale pourrait également causer des douleurs à l'extrémité de l'épaule, qui peuvent être soulagées en retirant le drain thoracique de quelques centimètres (Slinger $\mathrm{P}$, et McRae $\mathrm{K}$, communication personnelle). Ces résultats coïncident avec d'autres présentations de cas. ${ }^{7}$ En outre, bien que Scawn et coll. ${ }^{8}$ et Danelli et coll. ${ }^{9}$ aient montré une réduction considérable de la douleur à l'épaule post-thoracotomie à la suite d'une injection d'anesthésique local dans le panicule adipeux autour du nerf phrénique, un nombre significatif de leurs patients a continué à ressentir une douleur à l'épaule post-thoracotomie. Ainsi, il existe une possibilité que la douleur à l'épaule postthoracotomie provienne de sources autres que le nerf phrénique dans un certain sous-groupe de patients. Parmi les causes possibles, citons : des états arthritiques préexistants, la position du patient, une foulure aux articulations gléno-humérale ou acromio-claviculaire, un claquage musculaire par dessus l'omoplate ou le trapèze, l'extension de l'incision au-delà du niveau du bloc péridural, ou l'irritation au niveau de la plèvre pariétale provoquée par le drain thoracique.
Malgré la nature intense et pénible de ce syndrome postopératoire douloureux, il semble qu'il n'ait qu'une durée limitée. Il a été observé que la douleur à l'épaule post-thoracotomie diminue durant les quatre à six premières heures. ${ }^{11}$ Barak et coll. ${ }^{10}$ décrivent une douleur à l'épaule post-thoracotomie diminuant progressivement et allant au moins jusqu'au premier jour postopératoire, et Mac et coll. ${ }^{5}$ décrivent des scores de douleur diminuant progressivement pendant les 48 heures suivant la chirurgie. MacDougall et coll. ${ }^{11}$ ont suivi les patients de leur étude jusqu'à ce que la douleur disparaisse, ou jusqu'au congé de l'hôpital. L'intensité et la durée de la douleur ont toutes deux diminué de façon constante durant la période postopératoire. Ces chercheurs ont observé que la durée moyenne de la douleur à l'épaule post-thoracotomie atteignait quatre jours, et la durée moyenne de la douleur à l'épaule post-VTS était de moins de trois jours. ${ }^{11}$ Aucun patient ne présentait de douleur à l'épaule post-thoracotomie ou post-VTS au moment du congé de l'hôpital.

\section{La gabapentine et la douleur postopératoire}

Ces dernières années, plusieurs études ont été menées pour étudier la possibilité d'utiliser la gabapentine pour soulager la douleur postopératoire, voire pour prévenir les douleurs postopératoires chroniques. ${ }^{12,13}$ Il a été démontré que la gabapentine périopératoire est efficace pour réduire la douleur postopératoire provoquée par les chirurgies orthopédiques, gynécologiques, urologiques, thoraciques et ORL. ${ }^{12,13} \mathrm{Il}$ a également été montré qu'elle réduit la douleur postopératoire et diminue le recours aux opiacés pour le contrôle de la douleur postopératoire. ${ }^{12,13} \mathrm{Il}$ est intéressant de relever que la gabapentine, administrée avant l'opération (300 mg oralement), c'est-à-dire à une dose représentant un tiers de celle utilisée par Huot et coll. ${ }^{1}$, a efficacement réduit la douleur postopératoire après une cholécystectomie par laparoscopie. ${ }^{14}$ Cependant, dans cette vaste étude, le type de douleur (incision / viscéral / épaule) n'est pas décrit. En plus d'agir comme agent anti-nociceptif, la gabapentine réduit les nausées et vomissements postopératoires suivant une cholécystectomie par laparoscopie. ${ }^{12}$ En revanche, nous ne savons pas si cet effet est lié à une diminution des besoins en opiacés ou à un effet direct sur les médiateurs tels que les tachykinines.

À l'heure actuelle, le mécanisme précis qui a pour effet que la gabapentine réduit la douleur aiguë demeure inconnu. La gabapentine inhibe les canaux calciques dans les neurones hyper-excités, mais n'agit pas sur les neurones normaux. ${ }^{12,13,15}$ Peng et Wijeysundera ${ }^{12}$ suggèrent que cet inhibiteur de la sous- 
unité $\alpha-2-\delta$ des canaux calciques soit renommé agent «anti-sensibilisation» en plus de son statut d'agent anti-nociceptif. La gabapentine se lie spécifiquement à la sous-unité $\alpha-2-\delta$ du canal calcique dépendant d'un potentiel d'action dans le tissu neurologique, lequel $\mathrm{a}$, à son tour, au moins trois sous-unités $(\alpha-2-\delta-1$, $\alpha-2-\delta-2$ et $\alpha-2-\delta-3)$, chacune présentant diverses affinités de liaison avec la gabapentine. ${ }^{15}$ La gabapentine a un effet modéré sur la diffusion des neurotransmetteurs dans les cellules du néocortex et des nerfs périphériques, ${ }^{15}$ et elle a également un effet sur la modulation de plusieurs protéines kinases impliquées dans l'induction et le maintien des états hyperalgésiques. ${ }^{15}$

Pour résumer, les patients subissant des interventions ayant pour effet une irritation directe ou indirecte des fibres du nerf phrénique sont touchés par des taux élevés de douleur à l'épaule postopératoire. C'est particulièrement le cas chez les patients subissant des chirurgies thoraciques, qu'il s'agisse de procédures conventionnelles de thoracotomie ou de VTS. Cependant, au contraire de plusieurs formes de douleur postopératoire, cette douleur à l'épaule ne semble pas diminuer avec un traitement préventif à la gabapentine. En outre, bien que nous comprenions les sites de liaison de la gabapentine et certains de ses effets aussi bien à un niveau moléculaire que clinique, nombre de questions demeurent à ce jour sans réponse. L'effet de la gabapentine est-il, dans certains états douloureux postopératoires, médié par différentes sous-unités de la sous-unité $\alpha-2-\delta$ du canal calcique ? $\mathrm{Y}$-a-il une variation de la modulation post-translationnelle des neurotransmetteurs dans les différents états douloureux postopératoires? La douleur à l'épaule post-thoracotomie comporte-t-elle un aspect d'hyperalgésie ou de "remontée » qui serait sensible à une analgésie préventive ? L'éventail d'interactions potentielles entre les tissus et la gabapentine offre un terrain propice à la spéculation concernant les mécanismes sous-jacents par le biais desquels la gabapentine affecte de nombreux états douloureux postopératoires, mais n'affecte pas la douleur à l'épaule post-thoracotomie. Peut-être que cette 'intersection' d'un état douloureux postopératoire unique et d'un médicament présentant des mécanismes d'action nouveaux créera en fin de compte une fenêtre qui permettra de mieux comprendre pourquoi nous ressentons de la douleur après une chirurgie.

\section{References}

1 Huot MP, Chouinard P, Girard F, Ruel M, Lafontaine ER, Ferraro P. Gabapentin does not reduce postthoracotomy shoulder pain: a randomized, double- blind placebo-controlled study. Can J Anesth 2008; 55: 337-43.

2 Wills VL, Hunt DR. Pain after laparoscopic cholecystectomy. Br J Surg 2000; 87: 273-84.

3 Alexander JI. Pain after laparoscopy. Br J Anaesth 1997; 79: 369-78.

4 Bussieres J, St-Pierre A, Soucy J. Post-thoracotomy/ scopy shoulder pain : preliminary study. Can J Anesth 2007; 54: 44420 (abstract).

5 Mac TB, Girard F, Chouinard P, et al. Acetaminophen decreases early post-thoracotomy ipsilateral shoulder pain in patients with thoracic epidural analgesia: a double-blind placebo-controlled study. J Cardiothorac Vasc Anesth 2005; 19: 475-8.

6 MacDougall P. Postthoracotomy shoulder pain: diagnosis and management. Curr Opin Anaesthesiol 2008; 21: 12-5.

7 Bamgbade OA, Dorje P, Adbikary GS. The dual etiology of ipsilateral shoulder pain after thoracic surgery. J Clin Anesth 2007; 19: 296-8.

8 Scawn ND, Pennefather SH, Soorae A, Wang JX, Russell GN. Ipislateral shoulder pain after thoracotomy with epidural analgesia: the influence of phrenic nerve infiltration with lidocaine. Anesth Analg 2001; 93: 260-4.

9 Danelli G, Berti M, Casati A, et al. Ipisilateral shoulder pain after thoracotomy surgery: a prospective, randomized, double-blind, placebo-controlled evaluation of the efficacy of infiltrating the phrenic nerve with $0.2 \% \mathrm{wt} / \mathrm{vol}$ ropivacaine. Eur J Anaesthesiol 2007; 24: 596-601.

10 Barak M, Iaroshevski D, Poppa E, Ben-Nun A, Katz $\Upsilon$. Low-volume interscalene brachial plexus block for post-thoracotomy shoulder pain. J Cardiothorac Vasc Anesth 2007; 21: 554-7.

11 MacDougall $P$, Slinger $P$, McRae K, Ko R. A preliminary study of the incidence of post-thoracotomy shoulder pain. Anest Analg 2008; 106: S42 (abstract).

12 Peng PW, Wijeysundera DN, Li CC. Use of gabapentin for perioperative pain control: a meta-analysis. Pain Res Manag 2007; 12: 85-92.

13 Ho KY, Gan TJ, Habib AS. Gabapentin and postoperative pain-a systematic review of randomized controlled trials. Pain 2006; 126: 91-101.

14 Pandey CK, Priye S, Singh S, Singh U, Singh RB, Singh $P K$. Preemptive use of gabapentin significantly decreases postoperative pain and rescue analgesic requirements in laparoscopic cholecystectomy. Can J Anesth 2004; 51: 358-63.

15 Maneuf YP, Gonzalez MI, Sutton KS, Chung FZ, Pinnock RD, Lee K. Cellular and molecular action of the putative GABA-mimetic, gabapentin. Cell Mol Life Sci 2003; 60: 742-50. 